effects of the scattered light on Doppler shift measurements.

Theoreticians have been trying to explain the generation and maintenance of the observed equatorial accelerations by a mechanism which transports angular momentum towards the equator against the viscous dissipation. Now it seems well established that this mechanism is located in the convection zone and it is produced by the interaction of convection with rotation. Boussinesq models of convection in a rotating spherical shell are unsatisfactory, as they can hardly be applied to the Sun, due to the high stratified character of its convection zone. Inclusion of the compressibility appears to be essential for understanding, even in a qualitative way, the solar rotation mechanism.

The problem of the generation of the magnetic cycle cannot be dis- connected from the differential rotation problem. The mean magnetic field propagates in fact along the surfaces of isorotation, which therefore must be shaped by differential rotation in such a way that they allow for a correct field migration in order to display the observed Butterfly diagram.

Much has to be done in this field such as the inclusion of non-linear interactions in compressible models of solar rotation and also on dynamo models to take into account the back reaction of the magnetic field on the velocity field. But we probably are on the threshold of a much deeper understanding of the solar convection zone and solar activity, and by extrapolation we shall be able to add significantly to our knowledge of stellar structure and evolution.

\section{Paterno}

\title{
European Great Projects Errata and Addenda
}

\section{LEP Technology}

In last month's account of the Great European Projects, when writing of LEP, we confused the arguments relating to the choice of the ring magnets with those of the RF cavities. In a large diameter machine such as this $\mathrm{e}^{+} \mathrm{e}^{-}$ collider - $10 \mathrm{~km}$ for a maximum energy of $130 \mathrm{GeV}$ (cf. the ISR protonproton collider at CERN which for an energy of $31+31 \mathrm{GeV}$ has a diameter of $0.3 \mathrm{~km}$ ) the magnetic fields in the ring magnets are so modest there is no advantage to be gained in using superconducting techniques. However, the large powers needed in the RF cavities make superconducting techniques very interesting, provided the outstanding problems can be solved. LEP has, therefore, been designed with a magnet ring that will cater for the highest energies and with an RF system that can be stepped up in stages. To begin with, conventional RF cavities would be used, but later it is hoped that it would prove possible to go over to superconducting cavities.

It should also be noted that the design team has throughout been working on a single ring concept, only in the original proposal 32 circulating bunches were envisaged, leading to 64 intersection points, at only eight of which the beams were made to collide. In the later design there are, as previously explained, four bunches circu- lating in each direction which cross at eight intersections, all of which are used for experiments.

Cost of the second proposal would be around $1000 \mathrm{M} \mathrm{Sw.Fr}$. - unfortunately not $100 \mathrm{M} \mathrm{Sw.Fr}$. as was printed.

\section{Hadron Colliders}

It will have been evident that we also had printing problems with our antiproton sign and for clarity we repeat the CERN and NAL projects for hadron colliders:

NAL Tevatron development: proton-proton $1000+250 \mathrm{GeV}$ proton-antiproton $250+250 \mathrm{GeV}$ or with cooling $1000+1000 \mathrm{GeV}$ CERN modified SPS:

proton-antiproton: $540 \mathrm{GeV}$ centre of mass.

\section{European Synchrotron Radiation \\ Facility}

The Chairman of the Instruments and Beamlines sub-group for the study of the facility is Dr. B. Buras and not Furas as cited.

\section{IRAM}

Since the Rome Seminar the agreement between the CNRS and the MaxPlanck-Gesellschaft covering the setting up of the Institute for Radio Astronomy in the Millimetre Range has been signed. Provisional premises were to be available in Grenoble from 1 May.

\section{9}

\section{Hewlett-Packard Europhysics Prize}

The 1979 Hewlett-Packard Europhysics Prize has been awarded to a group of five physicists from several European countries for their contributions to our understanding of the interaction between surface acoustic waves and electrons and the practical application of the effects to a new range of devices of particular importance in information handling.

The winners of the award who will share equally the 20000 Sw.Fr. prize are:

Eric A. Ash, University College, London

Jeffrey $\mathrm{H}$. Collins, University of Edinburgh

Yuri V. Gulaev, Institute of Radio Engineering, Moscow Kjell A. Ingebrigtsen, Norwegian Institute of Technology, Trondheim

Edward G.S. Paige, Department of Engineering Science, Oxford University

Electron-phonon coupling is one of the fundamental interactions encountered in solids, governing many dissipative phenomena as well as superconductivity. The prize is awarded in particular for the work relating to electron-phonon coupling in piezo-semiconductors and layer structures, and methods for slowing down the acoustic waves so that they are a better match to the drifting electrons.

Previous winners of the Hewlett-Packard Europhysics Prize were:

1978 Zh. I. Alferov, A. F. Ioffe Physico-Technical Institute, Leningrad;

physics of heterojunctions.

1977 W. E. Spear, University of Dundee;

amorphous silicon devices. 1976 W. Helfrich, Freie Universität, Berlin;

physics of liquid crystals. 1975 V. S. Bagev, L. V. Keldysh and J.E. Pokrovsky, Lebedev Physical Institute, Moscow and M. Voos, Ecole Normale Supérieure, Paris;

condensation of excitons. 